\title{
LA POLITIQUE ÉTRANGÈRE FRANÇAISE FACE À LA RÉVOLUTION HONGROISE DE 1956
}

Gustáv D. Kecskès

Presses Universitaires de France | «Relations internationales »

2005/2 nº 122 | pages 87 à 103

ISSN 0335-2013

ISBN 9782130551980

Article disponible en ligne à l'adresse :

https://www.cairn.info/revue-relations-internationales-2005-2-page-87.htm

Distribution électronique Cairn.info pour Presses Universitaires de France.

(C) Presses Universitaires de France. Tous droits réservés pour tous pays.

La reproduction ou représentation de cet article, notamment par photocopie, n'est autorisée que dans les limites des conditions générales d'utilisation du site ou, le cas échéant, des conditions générales de la licence souscrite par votre établissement. Toute autre reproduction ou représentation, en tout ou partie, sous quelque forme et de quelque manière que ce soit, est interdite sauf accord préalable et écrit de l'éditeur, en dehors des cas prévus par la législation en vigueur en France. Il est précisé que son stockage dans une base de données est également interdit. 


\section{La politique étrangère française face à la révolution hongroise de $1956^{1}$}

1956 - Budapest : la date et le lieu s'associent dans l'esprit de beaucoup de Français. Le nom de la capitale hongroise est devenu l'un des symboles les plus douloureux des dernières années de la $\mathrm{IV}^{\mathrm{e}}$ République : après Budapest, de nombreux Français ont dû faire justice de leurs propres illusions sur les pays socialistes. Quant à la société française qui observait avec une sympathie particulière le déroulement rapide des événements et leur fin tragique, elle eut vite la possibilité d'exprimer sa compassion par des actes concrets : les milliers de réfugiés hongrois en France furent reçus à leur arrivée par des milliers de familles françaises, par des organisations laïques et religieuses, grâce aussi à une aide significative du gouvernement. Par la suite, l'opinion publique et la presse françaises se sont intéressées à la tragédie avec une intensité conforme à sa portée en automne 1956. Ils ont conservé le souvenir de la "Hongrie martyre " pendant les décennies où la vérité ne pouvait se faire jour en Hongrie... Toutefois, l'activité réelle du gouvernement français et ses motivations sur le plan intérieur et surtout international sont restées obscures jusqu'à nos jours. L'ouverture des archives aussi bien en Occident que dans l'ex-bloc soviétique, nous donne la possibilité de formuler une image beaucoup plus nuancée sur les répercussions de la révolution hongroise en France.

En constatant l'énormité des sources d'archives actuellement disponibles sur les échos politiques et sociaux français, nous avons décidé de nous concentrer sur la réaction de la diplomatie française à la révolution hongroise de 1956. Les autres composantes de notre étude - l'analyse de la politique intérieure française et des relations internationales - servent à en éclairer le contexte.

1. Cette étude résume les conclusions de ma thèse de doctorat intitulée La diplomatie française et la révolution hongroise de 1956 (sous la direction des $\mathrm{P}^{\mathrm{rs}}$ Mária Ormos et Élisabeth du Réau, Sorbonne Nouvelle, Paris III - Pécsi Tudományegyetem [Université de Pécs], 3 vol., Paris, 2003, 749 p.). Je remercie les institutions ayant soutenu financièrement mon travail, notamment, en France, le gouvernement français, l'Université de la Sorbonne-Nouvelle, Paris III, l'École des hautes études en sciences sociales, en Hongrie, l'Institut d'histoire de l'Académie hongroise des sciences, l'Université de Pécs, le Cold War History Research Center, la Fondation Soros (Budapest), l'Institut de 1956, l'Institut du $\mathrm{XX}^{\mathrm{e}}$ siècle, les Programmes nationaux de recherche fondamentale (OTKA, F-030125), la mairie du $\mathrm{IV}^{\mathrm{e}}$ arrondissement de Budapest et, en Belgique, la Communauté française de Belgique. 
Nous nous sommes donc efforcé de répondre à trois questions fondamentales: quels furent l'attitude et le comportement de la diplomatie française pendant la révolution hongroise de 1956 ? Comment s'articule cette réaction française par rapport à l'ensemble des relations internationales? Quel fut l'impact des événements hongrois de 1956 dans l'histoire des relations diplomatiques franco-hongroises ? La réponse à ces questions permet de comprendre de façon plus approfondie un épisode saillant de la guerre froide, le contexte international de la révolution hongroise de 1956, et la portée réelle d'un événement mondial très médiatique.

Du côté hongrois, après l'effondrement des régimes communistes en Europe du Centre et de l'Est en 1989-1990, les historiens commencèrent des recherches approfondies à partir des archives de 1956 et étudièrent ces événements à la lumière de préoccupations scientifiques et non plus exclusivement politiques. Des premiers résultats furent publiés. L'Institut pour l'histoire de la révolution hongroise de 1956, qui est né sous l'égide de l'Académie hongroise des sciences dès 1990, constitue un des piliers de cette activité. Dès le début, il eut pour objectif de mettre en évidence les dimensions internationales de la révolution. Jusqu'alors, les ouvrages importants paraissaient en Occident ${ }^{2}$. Or pour analyser les questions internationales, au cours des années 1960 et 1970, seules les sources secondaires ou publiques étaient disponibles: la presse, les décrets officiels et les mémoires. À partir de la deuxième moitié des années 1980, les fonds d'archives anglais, français et autres devinrent accessibles. La chute des systèmes communistes en Europe centrale et orientale en 1989-1990 rendit possible la découverte des secrets contenus dans les documents gouvernementaux et dans ceux des partis. En 1992-1993 les chercheurs purent accéder à une grande partie des principaux documents soviétiques.

En ce qui concerne l'étendue des dépouillements effectués : la partie la plus importante des sources soviétiques a déjà été publiée et analysée en détail $^{3}$, il en est de même pour les documents polonais ${ }^{4}$. Des chercheurs

2. Voici quelques exemples: François Fejto, La tragédie hongroise ou une révolution socialiste antisoviétique, préface de Jean-Paul Sartre, Paris, 1956 ; Paul E. Zinner, Revolution in Hungary, New York - London, 1962 ; Miklós Molnár, Victoire d’une défaite. Budapest 1956, Paris, 1968 ; Bill Lomax, Hungary 1956, London, 1976 ; Péter Gosztonyi, A magyar forradalom története [L’histoire de la révolution hongroise], München, 1981 ; Ferenc Fehér - Ágnes Heller, Hungary 1956 Revisited. The Message of a Revolution - a Quarter of a Century After, London-Boston, 1983 ; Federigo Argentieri - Lorenzo Gianotti, L'ottobre ungharese, Róma, 1985.

3. A «Jelcin-dosszié ". Szovjet dokumentumok 1956-ról [Le "Dossier Yeltsine ". Documents soviétiques sur 1956], sous la dir. d’Éva Gál, András Hegedus B., György Litván et János Rainer M., Budapest, 1993 ; Döntés a Kremlben, 1956. A szovjet pártelnökség vitái Magyarországról [La prise de décision du Kremlin. Les débats de la présidence du Parti soviétique à propos de la Hongrie], réuni par Vjacseszlav Szereda et János Rainer M., préface de Vjacseszlav Szereda, étude analytique de János Rainer M., Budapest, 1996 ; Hiányzó lapok 1956 történetébol. Dokumentumok a volt SZKP KB Levéltárából [Les pages manquantes sur l'histoire de 1956. Documents provenant des Archives du Comité central de l'ex-Parti communiste soviétique], sous la dir. de Vjacseszlav Szereda et Alekstandr Sztikalin, Budapest, 1993 ; Szovjet nagyköveti iratok Magyarországról, 1953-1956. Kiszeljov és Andropov titkos jelentései [Documents des ambassadeurs soviétiques sur la Hongrie, 1953-1956. Rapports secrets de Kiseliov et d'Andropov], réunis et préfacés par Magdolna Baráth, Budapest, 2002.

4. Az 1956-os magyar forradalom lengyel dokumentumai [Les documents polonais de la révolution hongroise de 1956], réunis, traduits et introduits par János Tischler, Budapest, 1996 (L'original polo- 
ont étudié les dossiers yougoslaves, est-allemands, tchécoslovaques et roumains $^{5}$, ainsi que les papiers américains ${ }^{6}$ et britanniques ${ }^{7}$. Des travaux existent déjà sur la perception de la révolution hongroise en Italie, en Suisse, en Autriche, en Belgique, au Pays-Bas et au Canada. Les synthèses parues à ce jour ne sont pas encore des monographies. Celle de Csaba Békés, publiée en 1996, est la plus significative ${ }^{8}$.

nais: Revolucja wegierska 1956 w polskich dokumentach, Varsovie, 1995); Tischler, Lengyelország és Magyarország a két nemzet sorsfordító történelmi eseményei idején, 1956-ban és 1980-1981-ben [La Pologne et la Hongrie à l'heure des événements décisifs des deux nations, en 1956 et en 1980-1981], thèse de doctorat, l’Université Eötvös Lóránd, Budapest, 2000.

5. Top secret. Magyar-jugoszláv kapcsolatok, 1956. Dokumentumok [Top secret. Les relations hungaro-yougoslaves, 1956. Documents], sous la dir. de József Kiss, Zoltán Ripp et István Vida, Budapest, 1995 ; Top secret. Magyar-jugoszláv kapcsolatok, 1956-1959. Dokumentumok [Top secret. Les relations hungaro-yougoslaves, 1956-1959. Documents], sous la dir. de József Kiss, Zoltán Ripp et István Vida, Budapest, 1997. Mihály Ruff, "Magyarország és az NDK kapcsolatairól » [Sur les relations de la Hongrie et de la RDA], Múltunk, n 2, 1995 ; Hope M. Harrison, "Az 1956-os magyar felkelés és a kelet-német pártpolitika " [La révolte hongroise de 1956 et la politique du Parti estallemand] et Christian F. Ostermann, "Kelet-Németország és a magyar forradalom » [L'Allemagne de l'Est et la révolution hongroise], in Évkönyv V. 1996/1997 [Almanach V. 1996/1997], Institut de 1956, Budapest, 1997 ; Tibor Hajdu, " "A magyar reakció népellenes puccsa”. A Csehszlovák KB 1956. december 5-6-i ülése a magyarországi eseményekrol» [Putsch antidémocratique de la réaction hongroise. La réunion du Comité central tchécoslovaque du 5-6 décembre 1956 sur les événements hongrois], in Évkönyv II. 1993 [Almanach II. 1993], Institut de 1956, Budapest, 1993 ; Archives nationales hongroises (Budapest): XXXII-16-a, Copie des documents provenant des archives de l'étranger relatives à la révolution hongroise de 1956, Tchécoslovaquie, 1955-1958 (la liste annotée des documents tchécoslovaques a été préparée par József Reiter en 1994) ; 1956. Explozia. Perceptii române, ingoslave si sovietice asupra evenimentelor din Polonia si Ungaria [1956. L'explosion. Les réactions roumaines, yougoslaves et soviétiques aux événements polonais et hongrois], les documents ont été édités par Corneliu Mihai Lungu et Mihai Retegan, Bucarest, 1996 ; Mihai Retegan, "A román kommunizmus és az 1956-os magyar és lengyel válság. A Politikai Bizottság titkos levéltárának újonnan feltárt dokumentumai » [Le communisme roumain et la crise hongroise et polonaise de 1956. Les documents récemment mis à jour des archives secrètes du Bureau politique], in Évkönyv V. 1996/1997, op. cit. ; Az 1956-os Intézet Budapest-Bukarest' 56 gyujteménye. Az 1956-os vonatkozású romániai dokumentumok online adattára [Collection Budapest-Bucarest de l'Institut de 1956. Documentation sur Internet des documents roumains relatifs à 1956], Institut de 1956, 2000, site Internet: http://www.rev.hu.

6. Foreign Relations of the United States, 1955-1957. Eastern Europe, vol. 25, sous la direction de John P. Glennon, en collaboration avec Edward C. Keefer, Ronald D. Landa, Stenley Shaloff, Washington, XXVI, 1990. "Amerikai követjelentések 1956-ból» [Rapports de l'ambassadeur américain de l'année 1956], présenté et préfacé par István Vida István, Társadalmi Szemle, n 2, 1992 ; Csaba Békés, "Az Egyesült Államok és a magyar semlegesség 1956-ban » [Les États-Unis et la neutralité hongroise en 1956], Évkönyv III. 1994 [Almanach III. 1994], Institut de 1956, Budapest 1994 ; Iratok a magyar-amerikai kapcsolatok történetéhez, 1957-1967. Dokumentumgyujtemény [Documents sur l'histoire des relations hungaro-américaines], sous la direction de László Borhi, Budapest, 2002.

7. "A budapesti angol követ titkos jelentése az 1956. Évi magyar forradalom okairól és következményeirol (1957. I. 3.)" [Rapport secret de l'ambassadeur anglais sur les causes et les conséquences de la révolution hongroise de 1956 - 3 janvier 1957], présenté par István Szaszkó, Levéltári Szemle, n 3 , 1990 ; «A budapesti angol követség 1956. Novemberi-decemberi jelentéseibol » [Rapports sélectionnés de la Légation anglaise à Budapest en novembre-décembre 1956], présenté par István Vida, Társadalmi Szemle, n 1, 1991 ; Csaba Békés, " A brit kormány és az 1956-os forradalom " [Le gouvernement britannique et la révolution de 1956], in Évkönyv I. 1992, Institut de 1956, Budapest, 1992 ; «A magyar kérdés az ENSZ-ben és a nyugati hatalmak titkos tárgyalásai, 1956. október 28. - november 4. Brit külügyi dokumentumok » [La question hongroise à l'ONU et les négociations secrètes des puissances occidentales, 28 octobre - 4 novembre 1956. Documents diplomatiques britanniques], présenté par Csaba Békés, in Évkönyv II. 1993, op. cit.; The Hungarian Revolution of 1956. Collections of Documents from the British Foreign Office, sous la dir. d'Éva Haraszti-Taylor, Nottingham, 1995.

8. Csaba Békés, Az 1956-os forradalom a világpolitikában. Tanulmány és válogatott dokumentumok [La révolution de 1956 dans la politique mondiale. Etude et documents sélectionnés], avec la collabo- 
En France, pendant les événements et par la suite, beaucoup d'articles et d'ouvrages furent publiés par des auteurs français et hongrois, à partir de postulats politico-idéologiques certes différents. François Fejto, journaliste d'origine hongroise de grande réputation, exigea, le premier parmi les intellectuels français, la vérité. Thomas Schreiber, envoyé spécial en Hongrie du quotidien Le Monde dans les jours de la révolution, se chargea directement de l'information sur les événements hongrois. Pierre Kende, Tibor Méray, Miklós Molnár, exilés en 1956, travaillèrent aussi beaucoup pour rassembler les souvenirs d'origine française. Les représentants les plus connus de l'intelligentsia française : Raymond Aron, Albert Camus, JeanPaul Sartre, Marcel Merleau-Ponty, Claude Lefort exposèrent aussi leurs opinions sur $1956^{9}$.

On recense un certain nombre de publications sur l'écho politique en France de la révolution hongroise ${ }^{10}$. La plupart de ces œuvres mettent en scène la réaction de la gauche française, surtout celle des intellectuels communistes. Ce fait s'explique probablement par la très forte influence du Parti communiste français sur la vie culturelle à cette époque. Les autres ouvrages analysent certains secteurs de la presse. Stéphane Dufoix qui a rédigé une thèse sur l'histoire de l'émigration politique d'Europe centrale après la Seconde Guerre mondiale en France, a déjà utilisé les archives pour étudier la question des réfugiés hongrois de 1956 et leur organisation $^{11}$. Du côté hongrois, on a assez peu publié sur l'écho en

ration de Katalin Somlai, Budapest, 1996; Csaba Békés, "The 1956 Hungarian Revolution and World Politics ", Cold War International History Project Working Paper, $\mathrm{n}^{\circ}$ 16, Woodrow Wilson International Center for Scholars, Washington, DC, 1996.

9. Voici quelques exemples: Raymond Aron, «De l'organisation de l'enthousiasme à l'organisation de la révolte", Le Figaro, 30 novembre 1956; Albert Camus, "Discours à la Salle Wagram ", prononcé à la Salle Wagram le 15 mars 1957, Franc-Tireur, 19 mars 1957 (Essais, Paris, 1965 (coll. "La Pléiade ")) ; Jean-Paul Sartre, "Le fantôme de Staline ", Les Temps modernes, n ${ }^{\text {os }} 129$, 130, 131, novembre-décembre 1956 -janvier 1957 ; Marcel Merleau-Ponty, "Réforme... ou maladie sénile du communisme?", L'Express, 23 novembre 1956 ; Claude Lefort, "L'Insurrection hongroise ", Socialisme ou Barbarie, décembre 1956 - février 1957.

10. Cf. travaux en France sous forme d'ouvrage : Stéphane Dufoix, Exil et politique. Le cas hongrois en France après 1956, mémoire de DEA sous la dir. de Marc Lazar, Université PanthéonSorbonne, Paris I, 1992-1993 ; Florance Grandsenne, Les intellectuels français face aux crises du communisme en Europe du Centre-Est. Perception et interprétation des mouvements et de leur répression (1956-1981), thèse de doctorat à l'Institut d'études politiques de Paris, sous la direction de Serge Berstein, soutenue en 1998, vol. 1, Budapest, 1956 ; Sarolta Klenjánszky, Les répercussions de la révolution hongroise de 1956 et de sa répression dans le Parti communiste français, mémoire de maitrise sous la dir. d'Olivier Faron, Université Lumière - Lyon II (Faculté de géographie, de tourisme et d'histoire), 2002 ; 1956. Le commencement de la fin, Actes du colloque "Budapest 1956-1996", Palais du Luxembourg, Paris, 28 et 29 octobre 1996, rédaction et présentation de François Fejto et Gilles Martinet, Paris, 1997 ; JeanFrançois Sirinelli, Intellectuels et passions françaises. Manifestes et pétitions au XX siècle, chap. VIII, "Un automne 1956 ", Paris, 1990 ; Olivier Tocqueville, L'Humanité face au coup de Prague de 1948 et à la révolution hongroise de 1956, mémoire de maitrise, 1980 ; Guy Turbet-Delof, La Révolution hongroise de 1956. Journal d'un témoin, préfacé par François Fejto, Paris, 1996; Christine Vignaud, L'opinion française face aux événements de l'Europe de l'Est de 1956, mémoire de maitrise sous la dir. de Robert Frank, 1986-1987.

11. Stéphane Dufoix, Exil et politique. Éléments pour une sociologie de la politique en émigration: l'exemple des Hongrois, des Polonais et des Tchécoslovaques en France de 1945 à nos jours, thèse pour le doctorat en science politique sous la dir. de Marc Lazar, Université Paris I, Panthéon-Sorbonne, Département de science politique, 1997, t. 1-2. 
France de la révolution. György Litván, ancien directeur scientifique de l'Institut de 1956, a recueilli des documents provenant du Quai d'Orsay et des archives du Parti communiste français, et en a exposé brièvement le contenu dans l'annuaire de l'Institut ${ }^{12}$. Les recherches menées par Zoltán Garadnai dans les Archives nationales hongroises éclairèrent aussi certains aspects des relations diplomatiques franco-hongroises de l'époque ${ }^{13}$. Aussi n'existait-il jusqu'à présent aucune étude de synthèse ni sur les répercussions politiques et sociales en France ni sur l'attitude de la diplomatie française face à cette crise. Notre étude - se basant en premier lieu sur les recherches d'archives - se charge surtout de cette dernière tâche ${ }^{14}$.

Conformément aux objectifs définis ci-dessus, le sujet de notre étude est la réaction de la diplomatie française à la révolution hongroise de 1956.

12. György Litván, «Francia dokumentumok 1956-ról» [Documents français sur 1956], Évkönyv III. 1994, op. cit.

13. Zoltán Garadnai, "A magyar-francia diplomáciai kapcsolatok története, 1945-1966 " [L'histoire des relations franco-hongroises, 1945-1966], Külpolitika, no 1-2, 2001.

14. La majeure partie de la thèse s'appuie sur les documents d'archives. Commençons par les sources françaises. Au ministère français des Affaires étrangères, nous avons travaillé sur les dossiers de la série Europe 1956-1960, notamment la sous-série Hongrie qui contient en abondance des documents relatifs à la révolution hongroise de 1956 (aspects politiques intérieurs et extérieurs, économiques, militaires et religieux). Sur la période 1961-1965, de nombreux documents sont déjà accessibles au sujet de l'émigration politique hongroise et des relations franco-hongroises. Les dossiers du cabinet du ministre et ceux du Secrétariat général renferment également des informations sur ce sujet. Les séries Nations Unies et Organisations internationales (NUOI) et le fonds du Service des Pactes se révèlent tout aussi précieuses ; les premières pour étudier la politique menée par la diplomatie française à l'ONU, l'autre pour examiner le comportement de la France à l'OTAN. Parmi les archives des autres institutions publiques françaises, citons les archives de la présidence de la République et du Conseil des ministres qui sont conservées aux Archives nationales. Les documents du ministère de l'Intérieur qu'on y trouve également, surtout les synthèses générales des rapports mensuels des préfets, représentent des matériaux de grande valeur. Les documents de l'Assemblée nationale et du Sénat (le Conseil de la République à l'époque), ainsi que ceux des services historiques des Armées ont utilement complété nos informations. Considérant les archives des partis politiques, nous avons mis à profit avant tout celles du parti socialiste (SFIO) et du Parti communiste français (PCF). Ces documents renseignent souvent sur les vrais motifs des comportements politiques et les débats éventuels au sein même des partis. Les papiers du Parti socialiste, qui était alors au pouvoir, comblent les lacunes des archives de certaines institutions publiques (par exemple les procès-verbaux des réunions du Conseil des ministres qui ne sont pas consultables). Les archives internes du PCF nous instruisent sur les stratégies au sein d'une direction soucieuse de surmonter l'une des plus grandes crises de son histoire. Faute du temps nécessaire, les archives religieuses repérées - catholiques, protestantes et juives - n'ont pu être dépouillées. Des recherches futures pourraient s'orienter en ce sens.

Parmi les archives du secrétariat international de l'OTAN, à Bruxelles, nous avons trouvé un grand nombre de documents issus des diverses instances de l'alliance. En les complétant par ceux du Service des pactes au Quai d'Orsay et par les télégrammes du représentant permanent belge à son ministre, on peut établir avec précision le rôle joué par l'OTAN dans la concertation des diplomaties non seulement à propos de la crise hongroise mais au sujet des rapports Est-Ouest en général. Les procès-verbaux des réunions du Conseil atlantique et les sources émanant des autres administrations nous renseignent sur l'évolution de l'attitude des pays membres et sur leur interaction. Cela nous montre dans quelle mesure la France a pu influencer ses partenaires de l'OTAN, et dans quelle proportion aussi l'alliance a exercé un contre-effet sur la politique française.

Enfin, les documents diplomatiques hongrois conservés aux Archives nationales hongroises, essentiellement les correspondances politiques entre le ministère des Affaires étrangères et la légation de Hongrie à Paris, ainsi que des notes internes rédigées par les services compétents de l'Administration centrale sur les contacts établis avec la légation de France à Budapest, apportent de nombreuses précisions sur le développement des relations diplomatiques franco-hongroises, évidemment à travers le prisme du point de vue officiel hongrois. 
Avant de développer le fond du sujet, nous présentons brièvement la politique du Quai d'Orsay envers la Hongrie avant l'explosion de la révolution, pendant et après les événements. En conséquence, nous allons mesurer les facteurs ayant exercé une influence sur la diplomatie française au cours du processus de prise de décision concernant la crise hongroise. Examinons d'abord les effets exercés par les antécédents et les événements mêmes sur la politique étrangère française et sa réaction à ces défis.

La libéralisation en Union soviétique après la mort de Staline, qualifiée de "dégel», étendue plus tard aux autres pays européens sous influence soviétique, fut favorablement accueillie par les diplomaties occidentales, y compris le Quai d'Orsay. Néanmoins celles-ci étaient parfaitement conscientes de leur marge de manœuvre étroite découlant du statu quo européen issu de la fin de la Seconde Guerre mondiale et de la guerre froide, notamment de son système bipolaire américanosoviétique. Elles ne voulurent pas accélérer le processus par des démarches trop énergiques. Cette attitude prudente et passive se révèle également après le 23 octobre, date du déclenchement de l'insurrection qui surprit autant Paris que les autres capitales occidentales.

Il est notoire que, le 26 juillet 1956, Gamal Abdel Nasser, président de l'Égypte, a annoncé la nationalisation du canal de Suez. La préparation minutieuse de la riposte militaire en collaboration avec la GrandeBretagne puis avec Israël, l'attaque israélienne du 29 octobre contre l'Égypte, suivi par le déclenchement de l'intervention franco-britannique sur Suez restreignirent davantage encore les possibilités d'actions, voire accrurent la passivité du gouvernement français dont l'attention se fixait évidemment sur le Moyen-Orient ${ }^{15}$. Au moment où les représentants du gouvernement exprimèrent leur sympathie envers la révolution et leur réprobation à l'encontre de la politique de répression soviétique menée en Hongrie, ils veillèrent à ce qu'aucune déclaration ne provoque une trop grande tension avec l'URSS. L'ONU était le lieu idéal pour une telle politique. La mise en avant de l'affaire hongroise aux Nations Unies paraissait nécessaire au Quai d'Orsay, non moins qu'au Foreign Office, désireux d'y allumer un contre-feu à cause de la réprobation internationale quasi générale de leur politique à Suez. Les deux alliés souhaitaient obtenir que l'Assemblée générale extraordinaire de l'ONU, convoquée le $1^{\mathrm{er}}$ novembre pour traiter la guerre de Suez, inscrive à son ordre du jour la cause hongroise. Ils espéraient ainsi diviser et en partie détourner l'attention de la crise du canal. Toutefois, aspirant à gagner la sympathie des pays du Tiers Monde, le gouvernement des États-Unis entrava ce projet. Dès lors la question de la révolution hongroise n'y fut officiellement débattue qu'après son étouffement par l'Armée soviétique le 4 novembre.

15. Les 22-24 octobre 1956 les négociations secrètes anglo-franco-israéliennes eurent lieu à Sèvres, au cours desquelles les parties définirent le scénario de la guerre de Suez. Sur les relations entre l'affaire de Suez et la révolution hongroise de 1956, voir Gusztáv Kecskés, "The Suez Crisis and the 1956 Hungarian Revolution", East European Quarterly, vol. XXXV, Spring 2001/1, p. 47-58. 
La politique de prudence et de non-intervention se manifesta également par le fait que la diplomatie française ne prit aucune initiative de nature à influencer les événements au sein du bloc soviétique. Les activités du Quai d'Orsay se bornèrent donc à la collecte, à la transmission et à l'interprétation des renseignements. Même si les diplomates français firent un bon travail en la matière, leurs conclusions ne pesèrent pas lourd dans la balance. Entrèrent surtout en ligne de compte le statu quo européen et Suez ${ }^{16}$.

La question qui se pose maintenant est de déterminer si, au-delà des considérations internationales, la politique intérieure et la société française ont exercé un quelconque effet sur l'attitude du gouvernement présidé par le socialiste Guy Mollet. À cette fin nous allons examiner brièvement les échos de la révolution hongroise en France ${ }^{17}$.

Dans le contexte de la guerre froide, sous la IV République, la politique extérieure fut inséparable des combats idéologiques internes ${ }^{18}$. C'est précisément le débat idéologique, notamment sur les valeurs du communisme confrontées avec celles du "monde libre », qui atteignit son point culminant avec la tragédie hongroise ${ }^{19}$. C'est pour cette raison que l'effet de la révolution hongroise toucha toutes les sphères de la vie sociale et politique française. La majeure partie des groupements politiques, sociaux et même culturels et religieux choisit alors de prendre publiquement position. Malgré les apparences, sous cet angle, l'affaire hongroise fut une question de politique intérieure plutôt qu'un problème international pour la France. La Hongrie était en dehors de son orbite et de sa zone d'intérêt. Sur le plan extérieur, la guerre d'Algérie, la crise de Suez et l'émergence de l'intégration européenne étaient des enjeux bien plus importants. L'extrême intensité de la réaction de la population ${ }^{20}$ et de la classe politique s'explique par plusieurs facteurs. Grâce à l'atmosphère politisée de la guerre froide, l'opinion publique fut très sensible à la lutte d'un peuple vivant de l'autre côté du rideau de fer. La presse renforça encore ces répercussions. La majeure partie de la population française s'est indignée de l'action brutale soviétique et de la position du PCF qui l'approuvait ${ }^{21}$. L'écrasement de la révolte hongroise par les Soviétiques,

16. La réaction de la politique étrangère française aux antécédents de la révolution hongroise et aux événements insurrectionnels - eux-mêmes est exposée dans la première partie de ma thèse (vol. I, p. 24-193). Les notes en bas de page revoient au détail des sources et de la littérature utilisées.

17. C'est dans la seconde partie de ma thèse que je présente les effets exercés par la révolution hongroise sur la société et la politique intérieure françaises (vol. I, p. 194-311).

18. Alfred Grosser, La $I V^{e}$ République et sa politique extérieure, Paris, 1972, p. 35. p. 80 .

19. Jean-Pierre Bernard, "Novembre 1956 à Paris", Vingtième siècle, no 30, avril-juin 1991,

20. Sur les réactions de l'opinion publique : Archives nationales (ci-après AN), Paris, Synthèses des rapports mensuels des préfets (ministère de l'Intérieur), F1 C III 1235: 1956, août à décembre, mois de novembre 1956.

21. Voir la déclaration du Bureau politique du Parti communiste français du 4 novembre 1956 dans L'Humanité, le 5 novembre 1956. Sur la crise et la tactique de la direction du Parti, cf. Philippe Robrieux, Histoire intérieure du Parti communiste, vol. 2 : 1945-1972. De la Libération à l'avènement de 
d'une part, et l'opération de Suez, de l'autre, fournirent occasion et arguments aux forces politiques anticommunistes et communistes dont la confrontation durait depuis longtemps. En outre l'affaire hongroise semblait permettre d'obtenir des gains politiques non négligeables. La politique de la SFIO et celle de la CGT-FO contre le PCF et la CGT furent à cet égard particulièrement significatives. En effet, les socialistes en condamnant très sévèrement les forces politiques communistes compromises par l'affaire hongroise, tâchaient d'accaparer leur électorat important. Voire ils ont fait des efforts pour séduire leurs militants ${ }^{22}$. Il est fort probable que l'on trouve là l'explication de la participation gouvernementale très marquée à l'action de solidarité envers les réfugiés hongrois. Le cabinet Guy Mollet utilisa donc la question hongroise comme un instrument de politique intérieure.

Il faut tout de même indiquer les limites de la répercussion des événements hongrois en France. Quelques semaines après l'intervention soviétique du 4 novembre, en fin décembre 1956, l'intérêt de l'opinion pour la tragédie hongroise avait diminué considérablement. Les forces politiques françaises ne s'efforçaient déjà plus de maintenir à l'ordre du jour la cause hongroise. Malgré la vivacité extraordinaire de la réaction, les échos de la crise hongroise en France n'entraînèrent pas de changement fondamental et durable ni dans l'opinion publique, ni dans la vie politique du pays. La direction thorézienne du PCF maîtrisa ses difficultés en quelques semaines. La position occupée par le Parti communiste dans la vie politique française ne changea guère: il garda en substance ses effectifs et sa force électorale.

On a montré ci-dessus comment le gouvernement français utilisa l'affaire de Hongrie dans la politique internationale afin de détourner l'attention sur la campagne de Suez en plaçant sur le devant de la scène la question hongroise. Avec l'instrumentalisation de cette cause, le cabinet Guy Mollet employa donc la même tactique en politique intérieure ${ }^{23}$. Par l'organisation au niveau national des collectes et des commémorations, ainsi que par la voie de la presse (surtout la radio) le gouvernement contribua grandement à la mobilisation de l'opinion publique française. Le programme le plus spectaculaire dans ce sens fut sans doute "une journée nationale en faveur de la population hongroise " pour le 18 novembre,

Georges Marchais, Paris, 1981. Les documents d'archives sur le sujet sont consultables aux archives du Parti communiste français, Paris, III, Archives "papier ", Archives de direction, archives du Secrétariat et du Bureau politique (1944-1971) : Décisions du Secrétariat (1956-1958); Décisions du Bureau politique (1956-1958). Enregistrements audio des réunions du Comité central du Parti communiste français de 1952 à 1962.

22. Office universitaire de recherche socialiste, Paris, Archives d'organisations, Parti socialiste SFIO (1944-1969) : sténographie des réunions du Comité directeur de la SFIO, t. 13, $1^{\text {er }}$ juillet $1956-$ 21 juin 1957.

23. Contribution de Robert Frank à la conférence intitulée Les intellectuels et l'opinion publique en France face à la révolution hongroise de 1956 les 17-19 octobre 1996 à l'Institut culturel français en Hongrie. Les interventions furent transcrites par Csaba Csapodi, 2000 (manuscrit déposé à la Médiathèque de l'Institut français de Budapest), p. 173. 
préparée avec précision par le ministère de l'Intérieur ${ }^{24}$. On ne trouve pas trace de l'effet exercé par la société et la politique intérieure sur le processus de prise de décision diplomatique en ce qui concerne la question hongroise. Le grand retentissement en France de la répression de l'insurrection hongroise fut à nouveau instrumentalisé quand les responsables du Quai d'Orsay la citèrent dans les discours prononcés devant différentes instances internationales, en particulier à l'Organisation des Nations Unies $^{25}$.

Le comportement du gouvernement français sur le plan international après l'écrasement de la révolution hongroise s'intégrait parfaitement dans la ligne générale de la politique occidentale. Le point principal de son activité restait la collecte, la transmission et l'interprétation des informations. Les démarches réelles et concrètes s'avérèrent rares dans cette période aussi. Énumérons et illustrons ainsi les facteurs qui ont pu influer sur l'attitude du Quai d'Orsay ${ }^{26}$.

L'explication fondamentale demeure la situation du pays par rapport au système bipolaire international. Vu le rattachement de la France au "camp occidental», il paraît normal que le gouvernement français ait exprimé en public sa réprobation contre l'Union soviétique et le gouvernement Kádár, arrivé au pouvoir en Hongrie par suite de l'étouffement de la révolution. Une telle attitude correspondait aux attentes du public. La diplomatie française concrétisa sa désapprobation dans les débats et des résolutions de l'Assemblée générale des Nations Unies, dans ses rapports bilatéraux avec l'Union soviétique et la Hongrie en se ralliant au boycott diplomatique des pays de l'OTAN, par l'accueil massif des réfugiés hongrois, et enfin par le soutien accordé à l'émigration politique hongroise. Toutefois les décideurs français ne voulaient pas aller au-delà. En somme, ils continuaient de respecter scrupuleusement les règles du jeu de la guerre froide, notamment l'inviolabilité tabou des sphères d'influence. Cette prudence se traduisit dans toute l'attitude et la démarche du Quai d'Orsay en la matière : il n'essaya pas d'exercer son influence sur le fond du déroulement des événements révolutionnaires hongrois, ni de ses suites. En conséquence la majeure partie des démarches diplomatiques du gouvernement français s'effectua hors de la zone touchée directement par la crise, aux Nations Unies et à l'OTAN ${ }^{27}$.

24. AN, rapports des préfets 1954-1959, F1 C III 1350 : Vœux et motions concernant la Hongrie (extraits de presse, secours à la Hongrie ; journée nationale du 18 novembre), 1956, dossier : Secours à la Hongrie, le 10 novembre 1956, télégramme du ministère de l'Intérieur (Affaires politiques) aux préfets de métropole y compris Seine, Journée nationale en faveur population hongroise.

25. Voir le discours de Christian Pineau, ministre des Affaires étrangères, prononcé devant l'Assemblée générale des Nations Unies le 19 novembre 1956; Archives du ministère des Affaires étrangères (ci-après AMAE), Paris, Nations Unies et Organisations internationales, boîte 243, n 2526 (19 novembre 1956) ; télégramme de V. Broustra, chef de la Mission permanente de la France auprès de l'Organisation des Nations Unies.

26. Je traite la politique étrangère française concernant l'écrasement de la révolution hongroise et l'établissement du régime Kádár dans la troisième partie de ma thèse (vol. II, p. 313-473).

27. Sur le rôle de l'Alliance atlantique à propos de la révolution hongroise à partir des documents des Archives du secrétariat international de l'OTAN voir Gusztáv Kecskés « The North Atlantic 
Aux Nations Unies où l'affaire hongroise fut à l'ordre du jour de l'Assemblée générale pendant plus de six ans, jusqu'à décembre 1962, le but visé par le gouvernement français devint de plus en plus évident : en collaboration avec les Américains, il souhaitait exercer un effet sur l'opinion mondiale, surtout sur les pays du Tiers Monde en leur montrant «le vrai visage" de l'Union soviétique en expansion. L'Alliance atlantique, outre les consultations entre les ministères des Affaires étrangères, servait à unifier la ligne de conduite des pays membres. Même si Paris participa avec zèle au boycott diplomatique contre Moscou et Budapest, il est caractéristique que les mesures de rétorsions de l'OTAN furent supprimées à l'égard de l'URSS, responsable première de la répression meurtrière, dès janvier 1957, sans tenir aucun compte des opinions occidentales ${ }^{28}$. On a en revanche tenu en quarantaine le gouvernement Kádár bien plus longtemps, tout en sachant qu'il s'agissait d'un pur gouvernement fantoche. Mais le maintien du boycott contre la Hongrie officielle paraissait naturellement beaucoup moins risqué et nuisible au Quai d'Orsay qui pouvait satisfaire ainsi aux attentes de son opinion publique. Malgré cette "réserve ", le gouvernement français ne voulait pas rompre. On a soigneusement évité les actions qui auraient pu mettre en danger le fonctionnement et/ou l'existence de la légation de France en Hongrie ${ }^{29}$. Dans la même logique, la mission diplomatique française attachait de l'importance aux rapports avec les résistants et les opposants au régime de Kádár surtout en vue de collecter des informations.

En ce qui concerne une véritable coopération sur le fond, nous avons mis au jour dans les mois suivant la révolution quelques rares cas isolés. La collaboration avec István Bibó, ministre d'État de la dernière formation gouvernementale pendant la révolution, fut probablement l'exemple le plus significatif dans son genre (Plan de compromis du règlement de la question hongroise). Cette prudence se révéla dans le traitement de l'émigration politique quand on lui souligna sans ambiguité que les autorités françaises s'opposaient au stockage et au transit d'armes sur le territoire national ${ }^{30}$. Un tel geste aurait pu entraîner un conflit ouvert avec l'Union soviétique. Enfin, le représentant français à l'OTAN contraria toute publicité tendant à montrer l'implication de l'Alliance ${ }^{31}$, non seulement durant la révolution

Treaty Organization and the Hungarian Revolution of 1956 ", The Ideas of the Hungarian Revolution, Suppressed and Victorious 1956-1999, sous la dir. de Lee W. Congdon et Béla K. Király, Boulder, Colorado - Highland Lakes, New Jersey, 2002, p. 112-141.

28. AMAE, Service des pactes, carton 210, $\mathrm{n}^{\circ} 50.012$ (9 janvier 1957) ; télégramme d'Alexandre Parodi, représentant permanent de la France auprès du Conseil de l'Atlantique Nord, Assistance des représentants des pays atlantiques aux réceptions soviétiques.

29. Note de la sous-direction d'Europe orientale du ministère des Affaires étrangères, Relations diplomatiques avec la Hongrie (début 1957 ?), Documents diplomatiques français (ci-après DDF), 1957, t. I ( $1^{\mathrm{er}}$ janvier - 30 juin), Paris, 1990 , p. 2-4.

30. AMAE, Europe, 1944-1960, 97 ; dossier A Kelet-európai Aligazgatóság feljegyzése a Külügymisztérium fotitkárához (1956. december 13.), création en Occident d'un "Comité national révolutionnaire " hongrois.

31. Ibid., Nations Unies et Organisations internationales, boîte $242, \mathrm{n}^{\circ} 50.408$ (27 octobre 1956) ; télégramme d'A. Parodi, représentant permanent de France au Conseil de l'OTAN. 
mais aussi en décembre 1956. En matière d'aide aux étudiants réfugiés, le Quai d'Orsay fit savoir qu' "il serait préférable d'éviter toute action directe de l'OTAN $»^{32}$. Le gouvernement français respectait par conséquent en permanence les règles non écrites du statu quo européen.

En liaison étroite avec le précédent, un deuxième facteur a influé sur le comportement diplomatique français: la prétention d'inscrire son action dans une coopération multilatérale resserrée, en particulier bien sûr avec les États-Unis et la Grande-Bretagne. Les décisions relatives aux rapports entretenus avec le gouvernement Kádár, l'accueil des réfugiés hongrois, l'action humanitaire en faveur de la population de Hongrie furent amplement discutés avec les alliés. Au cas où les autres pays de l'OTAN ne partageaient pas ses vues, le Quai d'Orsay renonçait : par exemple en ce qui concernait l'aide économique à la Hongrie lors des premiers mois de $1957^{33}$.

Quoique les attaques aériennes franco-britanniques contre l'Égypte aient commencé le 31 octobre, les parachutistes alliés sautèrent alors sur Port-Saïd le 5 novembre, et le débarquement franco-britannique s'opéra le 6, les gouvernements de Paris et de Londres, sous la pression américaine, durent alors se résigner à donner l'ordre de cessez-le-feu pour le 7 novembre à zéro heure, sans que l'objectif militaire soit complètement atteint. Ils étaient contraints d'accepter l'envoi en Égypte d'une force internationale d'urgence constituée par l'ONU. Les premiers casques bleus arrivèrent par avions le 15 novembre. Les effectifs de la force internationale atteignaient 3700 hommes en décembre 1956. Par contrecoup, l'évacuation des troupes franco-britanniques s'acheva le 22 décembre.

Une fois la crise de Suez passée, l'effet de ce facteur diminua graduellement sur la prise de décision française en cédant le pas au problème algérien qui persista jusqu'à 1962. La politique menée en Algérie réduisit dans une certaine mesure la marge de manœuvre de la France aux Nations Unies. Au total, on constate que le système bipolaire mondial n'a cessé de déterminer la diplomatie française vis-à-vis de la Hongrie, dans la période postérieure à la révolution. Il s'agit donc d'un facteur stratégique, tandis que les intérêts de la France au Moyen-Orient relevaient de facteurs tactiques et temporaires.

Après avoir examiné les divers aspects de la politique étrangère française envers la révolution hongroise de 1956, il est temps de répondre aux questions posées en introduction : comment a agi la diplomatie française face à la révolution hongroise de 1956 ? Comment s'intègre sa réaction à la révolte hongroise par rapport à l'histoire des relations internationales?

32. Archives du secrétariat international de l'OTAN (ci-après ASIO), Bruxelles, AC/52-R/67 (8 janvier 1957) ; procès-verbal de la réunion du Comité de l'information et des relations culturelles tenue au Palais de Chaillot, Paris, le 18 décembre 1956 à 15 heures.

33. AMAE, Service des pactes, carton 210, nº 50.132 (6 mars 1957) ; télégramme d'A. Parodi, représentant permanent de la France auprès du Conseil de l'Atlantique Nord, Aide à la Hongrie. 
Quel a été l'impact des événements hongrois de 1956 dans l'histoire des relations diplomatiques franco-hongroises?

La politique du Quai d'Orsay envers la Hongrie et les pays du bloc soviétique s'est caractérisée par une grande stabilité durant toute la période examinée. Son attitude se détermina tout d'abord en fonction des règles du système bipolaire de la guerre froide. Ce contexte engageait à la prudence, à une politique de non intervention, voire à la passivité. La politique du " relâchement " pacifique sur les pays satellites - c'est-à-dire l'incitation à mener une politique étrangère plus indépendante de l'Union soviétique et une politique intérieure de plus en plus libérale par des relations économique, politique et culturelle $e^{34}$ - qui fut une réponse, dans une certaine mesure, au "dégel» en Europe orientale, ne sortit jamais de ce cadre. Les points essentiels de la conception élaborée au printemps 1957 dans la politique étrangère des pays de l'OTAN s'esquissèrent avant les événements de Pologne et de Hongrie ${ }^{35}$. Ni la révolution hongroise, ni son écrasement n'apportèrent de modifications notables à cette ligne de conduite. Le gouvernement français ne planifia aucun projet en perspective de mesures plus énergiques. L'acceptation de la neutralité hongroise pendant la révolution ou la proposition de neutralisation de la Hongrie par Christian Pineau, ministre des Affaires étrangères français, le 18 décembre 1956 à l'Assemblée nationale ${ }^{36}$, n'entrent pas dans ce cadre. Le poids international de la France ne permettait aucune démarche d'envergure. De même les propos similaires de Jean Paul-Boncour, ministre de France en Hongrie, s'adressant à Chou En-laï, président du Conseil des ministres chinois en janvier 1957 à Budapest, n'étaient en fait qu'un simple coup d'essai ${ }^{37}$.

Les autres facteurs, à savoir l'impact de la crise de Suez et, à plus long terme, la guerre d'Algérie n'ont pas été sans influence, en particulier aux Nations Unies. Toutefois ces éléments n'exercèrent qu'un rôle provisoire et d'ordre tactique par rapport au statu quo européen. La réaction extrêmement intense de la société et de la vie politique françaises pèse peu dans la prise de décision du Quai d'Orsay. Certes les diplomates français ressentaient envers la Hongrie une compassion identique au reste de l'opinion publique (cf. Jean Laloy, Étienne Manac'h, Fernand Rouillon, Guy Turbet-Delof, etc. $)^{38}$, néanmoins la grande réaction émotive et les

34. Békés, Az 1956-os magyar forradalom a világpolitikában, op. cit., p. 77.

35. ASIO : C-M (56)110 (24 septembre 1956). Publié par «Az olvadás Kelet-Európában a Nyugat szemével. A NATO Nemzetközi Titkárságának belso dokumentuma » [Le dégel en Europe orientale du point de vue occidental. Un document interne du secrétariat international de l'OTAN], publié et traduit par Kecskés, Évkönyv VIII. 2000. Magyarország a jelenkorban [Almanach, VIII, 2000, La Hongrie au temps présent], Institut de 1956, Budapest, 2000, p. 369-391.

36. Journal officiel de la République française. Débats parlementaires, Assemblée nationale, compte rendu in extenso des séances, questions écrites et réponses des ministres à ces questions, troisième législature, session ordinaire de 1956-1957, $1^{\text {re }}$ séance du 18 décembre 1956, p. 6090.

37. Télégramme de Jean Paul-Boncour, ministre de France en Hongrie, $n^{\text {os }} 65$ à 69 (18 janvier 1957), DDF, 1957, t. I, op. cit., p. 104-105.

38. Sur la sympathie des diplomates français à l'égard de la révolution hongroise, voir Kecskés, "De l'autre côté du "rideau de fer"... La révolution hongroise de 1956 et la politique étrangère française à la lumière de quelques entretiens avec d'anciens diplomates ", Specimina Nova, 1999, p. 155-171. 
manifestations individuelles de sympathie ne pesèrent guère dans la balance finale.

La crise hongroise sortait de l'orbite française. On s'en rend compte au fait que les principaux foyers d'action de la politique étrangère française se sont déployés partout sauf à Budapest : à l'ONU, à l'OTAN, voire en France par l'accueil des réfugiés. Paris a donc concentré tous ses efforts là où il pouvait agir sans risquer la confrontation directe avec l'Union soviétique. Ainsi le boycott du gouvernement Kádár par les pays membres de l'OTAN permettait à Paris de critiquer la Hongrie au nom du libéralisme politique occidental, sans heurter de front Moscou. On scrutait naturellement les mouvements de l'URSS comme décisifs du point de vue de la détente internationale. Or la diplomatie française plaçait au rang des priorités la poursuite de la détente et du désarmement. En conséquence, le maintien du dialogue avec l'URSs fut jugé plus important que le soutien à l'émancipation des peuples d'Europe centrale et orientale. Cette attitude fut soulignée clairement en juin 1958, au moment de l'exécution d'Imre Nagy - Premier ministre hongrois pendant la révolution de 1956 - et de ses compagnons :

«... il convenait de ne pas mélanger les questions. Les conversations entre l'Est et l'Ouest se situaient sur un autre plan et l'enjeu en était trop grave pour laisser commander notre attitude à ce sujet par des mouvements émotionnels. L'opinion, qui comprendrait peut-être aujourd'hui si nous rompions les conversations de Moscou pour l'exécution de Nagy, nous le reprocherait sans doute dans quelques mois. Il fallait, certes, ne pas se précipiter, mais il ne fallait pas prendre des décisions irrévocables. (...) Nous étions donc partisans de ne pas rompre..." ",

explique Étienne de Crouy-Chanel, représentant permanent de la France au Conseil de l'otan le 20 juin $1958^{39}$.

D'après les sources disponibles, la question hongroise n'était pas prioritaire pour le gouvernement Guy Mollet, ni sur le plan intérieur, ni au point de vue international. Au cours des conseils des ministres durant la révolution hongroise, on n'aborda le sujet qu'une seule et unique fois. La première décision relative à la crise hongroise date du 7 novembre et porta sur les réfugiés. Par ailleurs, bien sûr, le gouvernement français s'est longuement saisi des intérêts nationaux qui l'affectaient directement: l'arraisonnement de l'Athos avec sa cargaison d'armes égyptiennes destinée aux rebelles algériens, l'interception de l'avion des chefs de FLN et les nouvelles de la guerre de Suez ${ }^{40}$. Si nous observons les contacts bilatéraux

39. AMAE, Services des Pactes, boîte 241, no 57 (20 juin 1958); télégramme d'Étienne de Crouy-Chanel, représentant permanent de France au Conseil de l'OTAN.

40. Je n'ai pas eu d'occasion d'étudier les procès-verbaux des réunions du Conseil des ministres. Je m'appuie sur les propos tenus par Patricia Gillet, conservateur compétent à la Section du XX $\mathrm{X}^{\mathrm{e}}$ siècle des Archives nationales (Paris), qui m’a communiqué certaines informations, et sur l'examen des cartons F60 2766 (Ordre du jour du Conseil des ministres, 1956-1958, dossier: janvier 1956décembre 1956) et F60 2772 (communiqués de presse des Conseils des ministres, dossier : janvier 1956 - décembre 1959). 
entre la France et ses alliés en novembre-décembre 1956, nous obtenons le même constat. Lors de ces négociations au plus haut niveau, l'affaire hongroise ne fut pas mentionnée, ou à peine. Lors de la rencontre célèbre de Guy Mollet-Konrad Adenauer du 6 novembre à Paris, le sujet principal fut l'intégration européenne ${ }^{41}$. À l'occasion de la visite de Hervé Alphand, ambassadeur de France aux États-Unis, au président Eisenhower, le 8 novembre, les partenaires soulignèrent surtout l'importance du renforcement de l'alliance entre les États-Unis, la Grande-Bretagne et la France après la crise de Suez, laquelle avait érodé la relation de confiance antérieure ${ }^{42}$. Enfin, pendant les négociations franco-britanniques, on traita presque uniquement de la guerre de Suez et de ses suites ${ }^{43}$. Dans les chancelleries, la question hongroise restait donc sans conteste en arrière-plan. On peut étendre le constat aux conseils ministériels de l'Union de l'Europe occidentale (UEO) du 10 décembre $^{44}$ et de l'OTAN, les 1114 décembre 1956 à Paris.

Le comportement et l'attitude du gouvernement français, qui viennent d'être analysés, s'intègrent parfaitement dans la ligne générale de la diplomatie occidentale. Les autres dirigeants aussi - contrairement à leurs opinions publiques - étaient pleinement conscients de l'étroitesse de leur marge de manœuvre face aux événements. L'idée d'une intervention militaire ou d'une action politique énergique contre le bloc soviétique ne leur vint pas à l'esprit. Ils voulaient également continuer le processus de détente dans les relations Est-Ouest et attachaient une grande importance au maintien du dialogue avec l'Union soviétique. A propos de la Hongrie, Paris s'est concerté en permanence avec ses partenaires au sein du forum de l'OTAN, et lors d'autres consultations bilatérales. Cela permit d'édifier une ligne diplomatique commune, prolongement aussi d'une situation géostratégique analogue. Les liens avec la Grande-Bretagne et les États-Unis restaient évidemment des plus étroits.

La révolution hongroise et sa répression n'ont ainsi pas provoqué de vrai conflit entre les blocs occidental et oriental, car l'URSS s'était contentée d'agir à l'intérieur de sa zone d'influence. Néanmoins les contemporains avaient l'impression que la crise hongroise et ses dangers résultaient de l'opposition idéologique entre les deux blocs. En effet, on pouvait tirer cette conclusion à partir de la propagande diffusée par les stations de radio américaines - la Radio Europe Libre et la Voix de l'Amérique

41. AMAE, Secrétariat général (1945-1966), "Entretiens et message », 3 septembre 1956 - septembre 1957 ; procès-verbal de l'entretien du 6 novembre 1956 entre G. Mollet et K. Adenauer (DDF, 1956, t. III : 24 octobre - 31 décembre, Paris, 1990, p. 231-238.)

42. Ibid., Secrétariat général (1945-1966), Suez, 82, n ${ }^{\text {os }} 7028$ à 7038 (8 novembre 1956) ; télégramme de Hervé Alphand, ambassadeur de France aux États-Unis (DDF, 1956, t. III, op. cit., p. 251-253).

43. Ibid., Secrétariat général (1945-1966), "Entretiens et message », 3 septembre 1956 - septembre 1957, n 278/SGL (4 décembre 1956) ; rapport de Jean Chauvel, ambassadeur de France en Grande-Bretagne.

44. Compte rendu de la séance du Conseil de l'UEO du 10 décembre 1956, DDF, 1956, t. III, op. cit., p. 512-518. 
en particulier - vers l'Europe de l'Est. Au cours de l'année 1956, il y fut suggéré que les États-Unis ne resteraient pas les bras croisés si le peuple d'un pays satellite s'insurgeait contre ses oppresseurs soviétiques... La réaction extrêmement intense de l'opinion publique occidentale renforça également l'appréciation qu'il s'agissait d'une circonstance très dangereuse. Il est important de souligner que la révolution hongroise ne s'assimile pas à une crise de ce genre, car en réalité elle ne risquait pas de provoquer une guerre mondiale. La situation était donc fort différente de la guerre de Corée par le passé, des deux crises de Berlin, de la crise des missiles à Cuba, de la guerre du Vietnam et de l'invasion soviétique de l'Afghanistan, plus tard, où les deux camps se sont effectivement opposés. La révolution hongroise de 1956 ressemblait plutôt à la révolte de Berlin-Est de 1953, au printemps de Prague de 1968 et aux événements de Pologne en 1980-1981, tous dans la sphère d'intérêt soviétique. Ces "crises " sont restées au niveau de la polémique, de la propagande et des opinions publiques, et ne troublèrent pas sérieusement les relations Est-Ouest ${ }^{45}$. On peut donc constater que, si la révolution hongroise augmenta provisoirement la tension entre les deux blocs, elle n'exerça pas d'effet notable au long terme sur leurs rapports réciproques ${ }^{46}$.

Observons, pour finir, l'influence de la révolution sur les relations franco-hongroises. L'amélioration progressive que l'on avait pu enregistrer dans les relations entre la France et la Hongrie en 1955-1956 fut non seulement arrêtée, mais brusquement inversée par le boycott diplomatique des pays de l'OTAN : "Sur le plan des représentations occidentales, la période de Rákosi est revenue ", se plaignit Henri Quioc, conseiller de la légation de France à Budapest en janvier $1957^{47}$. Néanmoins, on vit dès l'automne 1957 les prémices d'un réchauffement timide qui s'accélérera en l'espace d'un an, et, quelques années plus tard, dépassa considérablement le niveau de cordialité atteint la veille de la révolte. En avril 1959, un accord commercial fut signé ${ }^{48}$. En été 1960, on conclut un accord aérien franco-hongrois, et, en septembre, une ligne directe BudapestParis fut mise en service. En octobre 1961, un programme d'échange culturel fut signé à Paris. En mars-avril 1961, les représentants des partis parlementaires français les plus importants visitèrent la Hongrie. En conséquence un groupe d'amitié franco-hongrois fut créé à l'Assemblée nationale à Paris, ce qui provoqua la fondation d'un groupe similaire au Parlement hongrois. En avril 1963, une nouvelle délégation parlementaire française se rendit à Budapest. Les députés hongrois leur rendirent cette

45. Békés, "Hidegháború, enyhülés és az 1956-os magyar forradalom » [La Guerre froide, la détente et la révolution hongroise de 1956], Évkönyv V. 1996/1997, op. cit., p. 204 ; Békés, Az 1956-os magyar forradalom a világpolitikában, op. cit., p. 70-71.

46. Békés, Az 1956-os magyar forradalom a világpolitikában, op. cit., p. 70-71.

47. AMAE, Europe 1944-1960, Hongrie, dossier 89, n 157/EU (31 janvier 1957); rapport de Henri Quioc, conseiller de la légation de France en Hongrie, Situation et perspectives.

48. Ibid., Europe 1944-1960, Hongrie, dossier 121 (17 août 1959) ; note de la sous-direction d'Europe orientale, État des rapports entre la France et la Hongrie. 
visite en juillet 1963. Enfin, le 17 décembre 1963, on publia un communiqué élevant les représentations diplomatiques de deux pays au niveau d'ambassade ${ }^{49}$.

Le "dégel» graduel dans les rapports diplomatiques franco-hongrois peut s'expliquer par les facteurs suivants. Après le choc causé par l'intervention soviétique en Hongrie, le dialogue Est-Ouest s'intensifia au printemps 1957. La diplomatie française, comme celle de ses partenaires occidentaux, s'efforça d'appliquer la politique du "relâchement " communément admise à l'OTAN. A cette tendance générale s'ajouta la nouvelle politique d'ouverture du général de Gaulle envers l'Europe centrale et orientale ${ }^{50}$. La consolidation du régime Kádár rendit de plus en plus problématique à long terme le maintien de la réserve diplomatique vis-àvis de la Hongrie. De plus la diplomatie hongroise ne ménagea pas ses efforts pour normaliser ses rapports avec les pays de l'Alliance atlantique. Enfin, l'apaisement de la terreur et surtout la grande amnistie de 1963 aplanirent tous les obstacles à une normalisation complète et permirent le renforcement significatif des relations diplomatiques entre la Hongrie et la France.

En contemplant les rapports franco-hongrois sur plusieurs décennies, on peut constater que - contre toute apparence - la révolution hongroise de 1956 n'exerça pas une influence essentielle sur l'évolution de ces relations. Les propos du président de Gaulle tenus à l'occasion de la visite du nouvel ambassadeur hongrois en 1964 sont révélateurs à cet égard : "Il n'y avait, dans le passé, aucune cause de perturbation entre nos peuples qui pourrait entraver la progression constante dans les relations culturelles et économiques. " ${ }^{51} \mathrm{Il}$ est intéressant de noter que tous les domaines ne furent pas atteints au même degré : tandis que les rapports politiques, diplomatiques et culturels subissaient une fluctuation sinusoïdale, les relations économiques maintinrent un rythme plus stable ${ }^{52}$. Quoi qu'il en soit, malgré un boycott diplomatique de plusieurs années, les rapports franco-hongrois non seulement se normalisèrent, mais se consolidèrent dans les années 1960 à un niveau bien supérieur à celui d'octobre 1956.

L'impact de la révolution hongroise de 1956 dans l'histoire des relations franco-hongroises ne se retrouve guère dans la sphère diplomatique,

49. Archives nationales hongroises (ci-après ANH), Budapest, XIX-J-1-j, France, 1945-1964, carton 3, sz. n./1965 (23 avril 1965). Feljegyzés a magyar-francia kapcsolatok alakulásáról [Note sur le développement des relations franco-hongroises]. Cf. Garadnai, "A magyar-francia diplomáciai kapcsolatok története, 1945-1966", Külpolitika, no 1-2, 2001, op. cit., p. 115-119, 142-145.

50. Cf. Maurice Vaïsse, La grandeur. Politique étrangère du général de Gaulle, 1958-1969, Paris, 1998 (chap. 9 : «La politique de détente et l'ouverture à l’Est»), p. 413-451; Thomas Schreiber, Les actions de la France à l'Est ou les absences de Marianne, Paris, 2000 (chap. 2 : «Le rêve gaullien : détente, entente, coopération (1958-1969)»), p. 75-122.

51. ANH, XIX-J-1-j, France 1945-1964, carton 2, 004588/1/1964 (13 juillet 1964); rapport de Márton Valkó, ambassadeur de Hongrie en France, cité par Z. Garadnai, "A magyar-francia diplomáciai kapcsolatok története, 1945-1966 ", Külpolitika, no 1-2, 2001, op. cit., p. 119 et 145.

52. AMAE, Europe 1944-1960, Hongrie, dossier 107, (18 mai 1958) ; note de la sous-direction d'Europe orientale pour le secrétaire général, Questions hongroises. 
politique, économique ou culturelle. Les souvenirs mêlés de sentiments entretenus par la population française depuis presque un demi-siècle s'avèrent, eux, beaucoup plus durables. L'aide à la «Hongrie martyre » et les accueils de réfugiés ont compté pour beaucoup. De son côté, le peuple hongrois garde, lui, le souvenir de son abandon par l'Occident. À travers les études historiques toutefois, il doit prendre conscience que la majorité des Français étaient de leur côté, sinon par les armes, du moins avec le cœur.

Gusztáv KECSKÉS, Docteur en histoire, Sorbonne Nouvelle, Paris 3. 